\title{
HAUSDORFF DIMENSION IN INHOMOGENEOUS DIOPHANTINE APPROXIMATION
}

\author{
YANN BUGEAUD, DONG HAN KIM, SEONHEE LIM, AND MICHAE RAMS
}

\begin{abstract}
Let $\alpha$ be an irrational real number. We show that the set of $\varepsilon$-badly approximable numbers

$$
\operatorname{Bad}^{\varepsilon}(\alpha):=\left\{x \in[0,1]: \liminf _{|q| \rightarrow \infty}|q| \cdot\|q \alpha-x\| \geq \varepsilon\right\}
$$

has full Hausdorff dimension for some positive $\varepsilon$ if and only if $\alpha$ is singular on average. The condition is equivalent to the average $\frac{1}{k} \sum_{i=1, \cdots, k} \log a_{i}$ of the logarithms of the partial quotients $a_{i}$ of $\alpha$ going to infinity with $k$. We also consider one-sided approximation, obtain a stronger result when $a_{i}$ tends to infinity, and establish a partial result in higher dimensions.
\end{abstract}

\section{INTRODUCTION AND RESULTS}

A well-known result of Minkowski [15] asserts that, for every irrational real number $\alpha$ and every real number $x$, which is not of the form $m \alpha+n$ for integers $m, n$, there are infinitely many integers $q$ with

$$
|q| \cdot\|q \alpha-x\|<\frac{1}{4}
$$

where $\|z\|$ denotes the distance from $z$ to its nearest integer. This result was complemented by Kim [13] who proved that, for every irrational real number $\alpha$, the set of real numbers $x$ in $[0,1]$ such that $\liminf _{q \rightarrow \infty} q \cdot\|q \alpha-x\|=0$ has full Lebesgue measure. Subsequently, it has been proved in [2] (see also [17, 16]) that the complement set is large, namely, for every irrational real number $\alpha$, the set

$$
\operatorname{Bad}(\alpha):=\left\{x \in[0,1]: \liminf _{|q| \rightarrow \infty}|q| \cdot\|q \alpha-x\|>0\right\}
$$

has Hausdorff dimension 1. These results seem to indicate that all irrational real numbers behave in a same way, independently of their Diophantine properties.

However, if we refine our question and ask whether, for some positive real number $\varepsilon$, the set

$$
\operatorname{Bad}^{\varepsilon}(\alpha):=\left\{x \in[0,1]: \liminf _{|q| \rightarrow \infty}|q| \cdot\|q \alpha-x\| \geq \varepsilon\right\}
$$

is also of Hausdorff dimension 1, then we can distinguish $\alpha$ 's of distinct Diophantine properties. This is precisely the theme of this article.

1.1. Real numbers. It has been proved recently in [14] that, for almost every $\alpha$, we have

$$
\forall \varepsilon>0, \operatorname{dim}_{H} \operatorname{Bad}^{\varepsilon}(\alpha)<1 .
$$

In the same article, a sufficient condition which ensures (1.1), called heaviness (see (1.2) below), was given.

Our first main result gives necessary and sufficient conditions for (1.1) in two directions, one in terms of the convergents of $\alpha$ and the other in terms of singularity of $\alpha$.

Date: May 29, 2018.

2010 Mathematics Subject Classification: Primary 11K60 ; Secondary 28A80, 37E10. 
Throughout the article, for $\alpha=\left[a_{0} ; a_{1}, a_{2}, \cdots\right]:=a_{0}+\frac{1}{a_{1}+\frac{1}{a_{2}+\frac{1}{a_{3}+\cdots}}}$, its $k$-th convergent is denoted by

$$
\frac{p_{k}}{q_{k}}=\left[a_{0} ; a_{1}, \cdots, a_{k}\right]=a_{0}+\frac{1}{a_{1}+\frac{1}{a_{2}+\frac{1}{a_{3}+\cdots+\frac{1}{a_{k}}}}} .
$$

We call $\left(a_{k}\right)_{k \geq 0}$ the partial quotients of $\alpha$. An irrational real number $\alpha$ is called singular on average if, for every $c>0$,

$$
\lim _{N \rightarrow \infty} \frac{1}{N} \operatorname{Card}\left\{\ell \in\{1, \cdots, N\}:\|q \alpha\| \leq c 2^{-\ell} \text { has a solution with } 0<q \leq 2^{\ell}\right\}=1 .
$$

We establish in Section 4 that this property is equivalent to the fact that the sequence $\left(q_{k}^{1 / k}\right)_{k \geq 1}$ tends to infinity, stated in the following theorem.

Theorem 1.1. Let $\alpha$ be an irrational real number and, for $k \geq 1$, let $q_{k}$ denote the denominator of its $k$-th convergent. Then the following are equivalent.

(i) For some $\varepsilon>0$, the set $\operatorname{Bad}^{\varepsilon}(\alpha)$ has full Hausdorff dimension.

(ii) $\lim _{k \rightarrow \infty} q_{k}^{1 / k}=\infty$.

(iii) $\alpha$ is singular on average.

Moreover, if $\operatorname{dim}_{H} \operatorname{Bad}^{\varepsilon}(\alpha)=1$ for some $\varepsilon>0$, then so it is for every $\varepsilon$ in $\left(0,2^{-4} \cdot 3^{-3}\right)$.

The equivalence between Conditions (i) and (ii) is proved in Section 2.

According to [14], an irrational real number $\alpha=\left[a_{0} ; a_{1}, a_{2}, \ldots\right]$ is called heavy if, for every $\delta>0$, there exists $\eta>0$ such that

$$
\liminf _{N \rightarrow \infty} \frac{1}{N} \sum_{k=1}^{N} \max \left\{\log \eta a_{k}, 0\right\} \leq \delta .
$$

It was shown in [14] that if $\alpha$ is heavy, then $\operatorname{dim}_{H} \operatorname{Bad}^{\varepsilon}(\alpha)<1$ for all $\varepsilon>0$. Theorem 1.1 shows that the converse does not hold. Indeed, consider $\alpha=\left[0 ; a_{1}, a_{2}, \ldots\right]$ whose continued fraction expansion is defined by $a_{n}=1$, for $n$ not being an integer power of 2 , and by $a_{n}=3^{n}$ otherwise. Then, we observe that $\left(q_{k}^{1 / k}\right)_{k \geq 1}$ is bounded while $\alpha$ is not heavy.

Condition (ii) of Theorem 1.1 is satisfied when the partial quotients $\left(a_{i}\right)$ of $\alpha$ tends to infinity, in which case we can slightly strengthen the last statement of Theorem 1.1.

Theorem 1.2. Let $\alpha$ be an irrational real number whose sequence of partial quotients tends to infinity. Then, for every $\varepsilon<1 / 16$, we have $\operatorname{dim}_{H} \operatorname{Bad}^{\varepsilon}(\alpha)=1$.

Theorem 1.2 is proved in Section 2.

An analogue for one-sided approximation of Minkowski's result mentioned at the beginning of the introduction was obtained by Khintchine [11, who established that, for every irrational real number $\alpha$, every real number $x$, and every positive $\varepsilon$, there are infinitely many positive integers $q$ with

This statement motivates the study of the set

$$
q \cdot\|q \alpha-x\|<\frac{1+\varepsilon}{\sqrt{5}}
$$

$$
\operatorname{Bad}_{+}^{\varepsilon}(\alpha):=\left\{x \in[0,1]: \liminf _{q \rightarrow+\infty} q \cdot\|q \alpha-x\| \geq \varepsilon\right\} .
$$

Our main result in this direction is the following, more precise, theorem.

Theorem 1.3. Let $\alpha$ be an irrational whose sequence of partial quotients tends to infinity. Then, for every $\varepsilon<1 / 4$, we have

$$
\operatorname{dim}_{H}\left\{x \in[0,1] ; \liminf _{q \rightarrow \infty} q \cdot\|q \alpha-x\|=\varepsilon\right\}=1,
$$

while, for any $\varepsilon>1 / 4$, we have

$$
\left\{x \in[0,1] ; \liminf _{q \rightarrow \infty} q \cdot\|q \alpha-x\|=\varepsilon\right\}=\emptyset .
$$


It follows that $\operatorname{dim}_{H} \operatorname{Bad}_{+}^{\varepsilon}(\alpha)=1$, for $\varepsilon<1 / 4$, while $\operatorname{Bad}_{+}^{\varepsilon}(\alpha)$ is empty for any $\varepsilon>1 / 4$. It still remains to determine the Hausdorff dimension of $\operatorname{Bad}_{+}^{1 / 4}(\alpha)$. Theorem 1.3 is proved in Section 3.

The proof that (ii) implies (i) in Theorem 1.1 rests on arguments already present in [4, 2, 3], which extend to Diophantine approximation of matrices. We discuss this more general question in the following subsection.

1.2. Real matrices. If $\mathbf{x}$ is a (column) vector in $\mathbb{R}^{n}$, we denote by $|\mathbf{x}|$ the maximum of the absolute values of its coordinates. Define

$$
\|\mathbf{x}\|=\min _{\mathbf{z} \in \mathbb{Z}^{n}}|\mathbf{x}-\mathbf{z}| .
$$

Fix $m, n$ in $\mathbb{N}$ and let $A$ be an $n \times m$ real matrix. For $\varepsilon>0$, we define the set

$$
\operatorname{Bad}^{\varepsilon}(A):=\left\{\mathbf{x} \in[0,1]^{n}: \liminf _{\mathbf{q} \in \mathbb{Z}^{m}}|\mathbf{q}|^{m / n} \cdot\|A \mathbf{q}-\mathbf{x}\| \geq \varepsilon\right\}
$$

and we put

$$
\begin{aligned}
\operatorname{Bad}(A) & :=\bigcup_{\varepsilon>0} \operatorname{Bad}^{\varepsilon}(A)=\left\{\mathbf{x} \in[0,1]^{n}: \liminf _{\mathbf{q} \in \mathbb{Z}^{m}}|\mathbf{q}|^{m / n} \cdot\|A \mathbf{q}-\mathbf{x}\|>0\right\}, \\
\operatorname{Bad}^{\infty}(A) & :=\bigcap_{\varepsilon>0} \operatorname{Bad}^{\varepsilon}(A)=\left\{\mathbf{x} \in[0,1]^{n}: \liminf _{\mathbf{q} \in \mathbb{Z}^{m}}|\mathbf{q}|^{m / n} \cdot\|A \mathbf{q}-\mathbf{x}\|=+\infty\right\} .
\end{aligned}
$$

Theorem 1 of [2] asserts that

$$
\operatorname{dim}_{H} \operatorname{Bad}(A)=n .
$$

Before stating our main result in higher dimension, let us introduce some definitions and explain the general principle behind the proof of (1.3). Dirichlet's Theorem implies that, for any $X>1$, the inequalities

$$
\|A \mathbf{x}\| \leq X^{-m / n} \quad \text { and } \quad 0<|\mathbf{x}| \leq X
$$

have a solution $\mathbf{x}$ in $\mathbb{Z}^{m}$. The following definition of singularity goes back to Khintchine [12].

Definition 1.4. Let $m, n$ be positive integers and $A$ a $n \times m$ real matrix.

(1) The matrix $A$ is called singular if, for every $c>0$, the inequalities (1.4)

$$
\|A \mathbf{x}\| \leq c X^{-m / n} \text { and } 0<|\mathbf{x}| \leq X
$$

have a solution $\mathbf{x}$ in $\mathbb{Z}^{m}$ for any sufficiently large $X$.

(2) The matrix $A$ is called singular on average if, for every $c>0$, $\lim _{N \rightarrow \infty} \frac{1}{N} \operatorname{Card}\left\{\ell \in\{1, \cdots, N\}:\right.$ the inequalities (1.4) have a solution for $\left.X=2^{\ell}\right\}=1$.

(3) The matrix $A$ is called very well uniformly approximable if there exists a positive $\varepsilon$ such that the inequalities

$$
\|A \mathbf{x}\| \leq X^{-\varepsilon-m / n} \quad \text { and } \quad 0<|\mathbf{x}| \leq X
$$

have a solution $\mathbf{x}$ in $\mathbb{Z}^{m}$ for any sufficiently large $X$.

If $n=m=1$ and $A=(\alpha)$, then we say that $\alpha$ is singular (resp., singular on average, very well uniformly approximable) if $(\alpha)$ has this property.

Remark. As far as we are aware, the notion of singular on average matrices has been introduced in [9], motivated by the dynamical notion of points which escape on average under the action of a semigroup. The terminology very well uniformly approximable refers to the hat exponents introduced in 3 .

Remark. If the subgroup $G_{A}=A \mathbb{Z}^{m}+\mathbb{Z}^{n}$ of $\mathbb{R}^{n}$ has rank $\operatorname{rk}_{\mathbb{Z}}\left(G_{A}\right)$ smaller than $m+n$, then there exists arbitrarly large $\mathbf{x}$ in $\mathbb{Z}^{m}$ such that $\|A \mathbf{x}\|=0$. Throughout the paper, we consider only matrices $A$ for which $\operatorname{rk}_{\mathbb{Z}}\left(G_{A}\right)=m+n$. 
When $m=n=1$, using the theory of continued fractions, one can prove that, for any irrational real number $\xi$, there are arbitrarily large integers $X$ such that the inequalities

$$
\|q \xi\| \leq \frac{1}{2 X} \quad \text { and } \quad 0<q \leq X
$$

have no integer solutions; see [10] or Proposition 2.2.4 of [1]. Consequently, there are no singular real irrational numbers and, a fortiori, no very well uniformly approximable real irrational numbers neither. However, there do exist real irrational numbers which are singular on average; see Section 4.

The proof of (1.3) is based on a transference argument of [3] which relates classical Diophantine approximation properties of a matrix to its uniform inhomogeneous aproximation properties. In particular, the Theorem of [3] implies that for every very well uniformly approximable matrix $A$, the set $\operatorname{Bad}^{\infty}(A)$ has full Lebesgue measure. Note also that for every singular matrix $A$, the set $\operatorname{Bad}^{\infty}(A)$ has full Hausdorff dimension. This was proven by Moshchevitin [16] and, independently, by Einsiedler and Tseng [6]. Note that it follows from Minkowski's theorem quoted in Section 1 that, for every real irrational number $\alpha$, the set $\operatorname{Bad}^{\infty}((\alpha))$ is empty.

We can partially extend Theorem 1.1 to the case $(n, m) \neq(1,1)$. To describe our result, we first need to define the notion of best approximation vectors associated to a matrix $A=\left(\alpha_{i, j}\right)$. We denote by

$$
M_{j}(\mathbf{y})=\left({ }^{t} A \mathbf{y}\right)_{j}=\sum_{i=1}^{n} \alpha_{i, j} y_{i}, \quad \mathbf{y}={ }^{t}\left(y_{1}, \ldots, y_{n}\right), \quad(1 \leq j \leq m)
$$

the linear forms determined by the columns of $A$ and we set

$$
M(\mathbf{y})=\left\|^{t} A \mathbf{y}\right\|=\max _{1 \leq j \leq m}\left\|M_{j}(\mathbf{y})\right\| .
$$

Observe that the quantity $M(\mathbf{y})$ is positive for all non-zero integer $n$-tuples $\mathbf{y}$, since we have assumed that $\operatorname{rk}\left(G_{A}\right)=m+n$. Thus, we can build inductively a sequence of integer vectors

$$
\mathbf{y}_{i}={ }^{t}\left(y_{i, 1}, \ldots, y_{i, n}\right), \quad(i \geq 1),
$$

called a sequence of best approximations related to the linear forms $M_{1}, \ldots, M_{m}$ and to the supremum norm, which satisfies the following properties:

(1) Setting, $\left|\mathbf{y}_{i}\right|=Y_{i}$ and $M_{i}=M\left(\mathbf{y}_{i}\right)$, we have

$$
1=Y_{1}<Y_{2}<\cdots \text { and } M_{1}>M_{2}>\cdots,
$$

(2) $M(\mathbf{y}) \geq M_{i}$ for all non-zero integer vectors $\mathbf{y}$ of norm $|\mathbf{y}|<Y_{i+1}$.

We start the construction with a smallest minimal point $\mathbf{y}_{1}$ in the sense of [5], satisfying $Y_{1}=\left|\mathbf{y}_{1}\right|=1$ and $M(\mathbf{y}) \geq M\left(\mathbf{y}_{1}\right)=M_{1}$ for any integer point $\mathbf{y}$ in $\mathbb{Z}^{n}$ with norm $|\mathbf{y}|=1$. Suppose that $\mathbf{y}_{1}, \ldots, \mathbf{y}_{i}$ have already been constructed in such a way that $M(\mathbf{y}) \geq M_{i}$ for all non-zero integer point $\mathbf{y}$ of norm $|\mathbf{y}| \leq Y_{i}$. Let $Y$ be the smallest positive integer $>Y_{i}$ for which there exists an integer point $\mathbf{z}$ verifying $|\mathbf{z}|=Y$ and $M(\mathbf{z})<M_{i}$. The integer $Y$ does exist by Dirichlet box principle since $M_{i}>0$. Among those points $\mathbf{z}$, we select an element $\mathbf{y}$ for which $M(\mathbf{z})$ is minimal. We then set

$$
\mathbf{y}_{i+1}=\mathbf{y}, \quad Y_{i+1}=Y, \text { and } M_{i+1}=M(\mathbf{y}) .
$$

The sequence $\left(\mathbf{y}_{i}\right)_{i \geq 1}$ obtained in this way clearly satisfies the desired properties.

Furthermore, as established along the proof of Lemma 1 of [3], we have

$$
Y_{i+3^{m+n}} \geq 2 Y_{i+1}, \quad i \geq 1 .
$$

In the case $m=n=1$, the sequence of best approximations coincides with the sequence of denominators of convergents.

The implication (ii) $\Rightarrow$ (i) of Theorem 1.1 extends as follows. 
Theorem 1.5. Let $A$ be an $n \times m$ matrix and $\left(\mathbf{y}_{k}\right)_{k \geq 1}$ a sequence of best approximation vectors associated to $A$. If $\left|\mathbf{y}_{k}\right|^{1 / k}$ tends to infinity with $k$, then there exists a positive real number $\varepsilon$ such that

$$
\operatorname{dim}_{H} \operatorname{Bad}^{\varepsilon}(A)=n .
$$

If, furthermore, $\left|\mathbf{y}_{k+1}\right| /\left|\mathbf{y}_{k}\right|$ tends to infinity, then $\varepsilon$ can be taken to be any positive real number less than $(4 n)^{-1}(4 m)^{-m / n}$.

We do not know whether the rest of Theorem 1.1 extends to matrices, that is, whether the properties ' $A$ is singular on average' and ' $Y_{k}^{1 / k}$ tends to infinity' coincide in dimension $m \times n$ with $(m, n) \neq(1,1)$ and also if these conditions are equivalent to the existence of $\varepsilon>0$ with $\operatorname{dim}_{H} \operatorname{Bad}^{\varepsilon}(A)=n$.

\section{BADly APPROXIMABLE NUMBERS AND THE CONVERGENTS}

In this section, we prove Theorem 1.1 (i) $\Leftrightarrow$ (ii) and Theorem 1.2 ,

2.1. Inhomogeneous approximation using homogeneous approximation. In this subsection, we use a result concerning the Hausdorff dimension in homogeneous Diophantine approximation to prove Theorem 1.2 and implication (ii) $\Longrightarrow$ (i) of Theorem 1.1

We start with a corollary of a theorem of Erdős and Taylor [7, of which we give a proof for the sake of completeness.

Theorem 2.1. Fix $0<\delta<1 / 2$. Let $\left(n_{k}\right)_{k \geq 1}$ be an increasing sequence of integers such that $n_{k+1} / n_{k} \geq 4 /(1-2 \delta)$ for $k$ sufficiently large and $\lim n_{k}^{1 / k}=\infty$. The set

$$
\mathcal{S}_{\delta}=\left\{x \in[0,1] \text { : there exists } k_{0}(x) \text { such that }\left\|n_{k} x\right\|>\delta \text { for all } k \geq k_{0}(x)\right\} .
$$

has Hausdorff dimension 1. Moreover, if $\lim n_{k+1} / n_{k}=\infty$, then $\operatorname{dim}_{H} S_{\delta}=1$ for any $\delta$ in $(0,1 / 2)$.

Proof. Let $\delta$ be real with $0<\delta<1 / 2$. We consider the Cantor set $\mathcal{S}_{\delta}:=\cap_{k} E_{k, \delta}$, where

$$
E_{k, \delta}=[0,1] \cap \bigcup_{0 \leq j \leq n_{k}}\left[\frac{j+\delta}{n_{k}}, \frac{j+1-\delta}{n_{k}}\right] .
$$

The length of the intervals composing $E_{k, \delta}$ is equal to $(1-2 \delta) n_{k}^{-1}$. The distance between two intervals in $E_{k, \delta}$ is $2 \delta n_{k}^{-1}$. An interval composing $E_{k, \delta}$ contains at least $(1-2 \delta) n_{k+1} / n_{k}-2$ intervals composing $E_{k+1, \delta}$. For $k$ large enough, since $n_{k+1} \geq 4 n_{k} /(1-2 \delta)$, we see that any interval composing $E_{k, \delta}$ contains at least 2 intervals composing $E_{k+1, \delta}$. We are in position to apply the mass distribution principle. By Example 4.6 of [8], we obtain that

$$
\operatorname{dim}_{H} \mathcal{S}_{\delta} \geq \liminf _{k \rightarrow \infty} \frac{\log \left(m_{1} m_{2} \cdots m_{k-1}\right)}{-\log \left(m_{k} \varepsilon_{k}\right)},
$$

where $m_{k}$ is the smallest number of intervals of $E_{k, \delta}$ in each interval of $E_{k-1, \delta}$, and $\varepsilon_{k} \geq 2 \delta / n_{k}$ is the minimal distance between intervals of $E_{k, \delta}$. We check for sufficiently large $k$

$$
m_{k} \geq \frac{(1-2 \delta) n_{k}}{n_{k-1}}-2=\frac{(1-2 \delta) n_{k}}{2 n_{k-1}} .
$$

Thus we have

$$
\operatorname{dim}_{H} \mathcal{S}_{\delta} \geq \liminf _{k \rightarrow \infty} \frac{\log n_{k-1}+k \log ((1-2 \delta) / 2)}{\log n_{k-1}-\log (\delta(1-2 \delta))} .
$$

Since $\lim n_{k}^{1 / k}=\infty$, under the assumption $n_{k+1} \geq 4 n_{k} /(1-2 \delta)$ for $k$ large enough, we have $\operatorname{dim} \mathcal{S}_{\delta}=1$.

If $n_{k+1} / n_{k}$ tends to infinity, then for any given $\delta$ in $(0,1 / 2)$, the assumption $n_{k+1} \geq 4 n_{k} /(1-$ $2 \delta$ ) is satisfied for $k$ large enough. This shows that $\operatorname{dim} \mathcal{S}_{\delta}=1$. 
Proof of Theorem 1.2. We apply the second assertion of Theorem 2.1 to prove Theorem 1.2. Let $\alpha$ be an irrational number and $\left(q_{n}\right)_{n \geq 1}$ the sequence of denominators of its convergents. Assume that $q_{n+1} / q_{n}$ tends to infinity (equivalently, that $a_{n}$ tends to infinity). Let $k$ be an integer. Let $x$ be in $(0,1)$ and observe that, for every integer $y$, we have

$$
\|y x\| \leq|y| \cdot\|\alpha k-x\|+|k| \cdot\|\alpha y\| .
$$

Let $\delta$ with $0<\delta<1 / 2$. Assume that $|k|$ is large and let $\ell$ be the integer with

$$
q_{\ell} \leq \frac{2}{\delta}|k|<q_{\ell+1} .
$$

Assume that $x$ is in $\mathcal{S}_{\delta}$. Letting $y=q_{\ell}$ in (2.1), we have

This gives

$$
\left|q_{\ell}\right| \cdot\|\alpha k-x\| \geq\left\|q_{\ell} x\right\|-|k| \cdot\left\|\alpha q_{\ell}\right\| \geq \delta-\frac{|k|}{q_{\ell+1}}=\frac{\delta}{2} .
$$

$$
|k| \cdot\|\alpha k-x\| \geq \frac{\delta^{2}}{4}
$$

Since $\delta$ can be chosen arbitrarily close to $1 / 2$, the theorem is proved.

Theorem 2.2. Let $\alpha$ be a real number for which $q_{k}^{1 / k}$ tends to infinity. We have

$$
\operatorname{dim}_{H} \operatorname{Bad}^{1 /\left(2^{4} \cdot 3^{3}\right)}(\alpha)=1 .
$$

Proof. We first claim that for each $R>1$ there exists an increasing function $\varphi: \mathbb{Z}_{\geq 1} \rightarrow \mathbb{Z}_{\geq 1}$ satisfying $\varphi(1) \geq 1$ and, for any integer $i \geq 2$,

$$
q_{\varphi(i)} \geq R q_{\varphi(i-1)} \text { and } \quad q_{\varphi(i-1)+1} \geq q_{\varphi(i)} / R .
$$

The function $\varphi$ is constructed in the following way. Let

$$
\mathcal{J}_{0}=\left\{j: q_{j+1} \geq R q_{j}\right\},
$$

which is an infinite set since $q_{k}^{1 / k}$ tends to infinity. Let $\varphi(1)$ be the smallest element of $\mathcal{J}_{0}$. Suppose that we have defined $\varphi$ up to $\varphi(h)$ in $\mathcal{J}_{0}$. We will define $\varphi(h+1), \cdots, \varphi\left(h^{\prime}\right)$ for some $h^{\prime}$ which we will determine shortly. Define $\varphi\left(h^{\prime}\right)$ to be the smallest element of $\mathcal{J}_{0}$ greater than $\varphi(h)$.

Define $\varphi\left(h^{\prime}-1\right)$ to be the largest index $t>\varphi(h)$ for which $q_{\varphi\left(h^{\prime}\right)} \geq R q_{t}$. We let $\varphi\left(h^{\prime}-2\right)$ be the largest index $t>\varphi(h)$ for which $q_{\varphi\left(h^{\prime}-1\right)} \geq R q_{t}$, and so on until it does not exist any index $t$ as above. Let us say that we have just defined $\varphi\left(h^{\prime}\right), \varphi\left(h^{\prime}-1\right), \ldots, \varphi\left(h^{\prime}-h_{0}\right)$. Define $h^{\prime}=h_{0}+h+1$. It is easy to check that the inequalities (2.2) are satisfied for $i=h+1, \ldots, h_{0}+h+1$. The claim follows.

For a given $0<\delta<1 / 2$, let $R=\frac{4}{1-2 \delta}$. As $\varphi$ is increasing, $q_{\varphi(k)}^{1 / k}$ tends to infinity. Thus, we can apply Theorem 2.1 to the sequence $n_{k}=q_{\varphi(k)}$ to obtain a set $\mathcal{S}_{\delta}$. For $x$ in $S_{\delta}$, we argue as in the proof of Theorem 1.2 .

Let $k$ be an integer. Fix a positive real number $M$ and let $\ell$ be the integer with

$$
q_{\varphi(\ell)} \leq M|k|<q_{\varphi(\ell+1)} .
$$

Note that, by (2.2), we have

$$
\left\|\alpha q_{\varphi(\ell)}\right\| \leq q_{\varphi(\ell)+1}^{-1} \leq R q_{\varphi(\ell+1)}^{-1} .
$$

Thus by (2.1), if $x$ is in $\mathcal{S}_{\delta}$, then

$$
\begin{aligned}
\left|q_{\varphi(\ell)}\right| \cdot\|\alpha k-x\| & \geq\left\|q_{\varphi(\ell)} x\right\|-|k| \cdot\left\|\alpha q_{\varphi(\ell)}\right\| \\
& >\delta-R \cdot|k| \cdot q_{\varphi(\ell+1)}^{-1} \geq \delta-\frac{R}{M}
\end{aligned}
$$

This gives

$$
|k| \cdot\|\alpha k-x\| \geq \frac{1}{M} q_{\varphi(\ell)}\|\alpha k-x\| \geq\left(\delta-\frac{R}{M}\right) \frac{1}{M}
$$




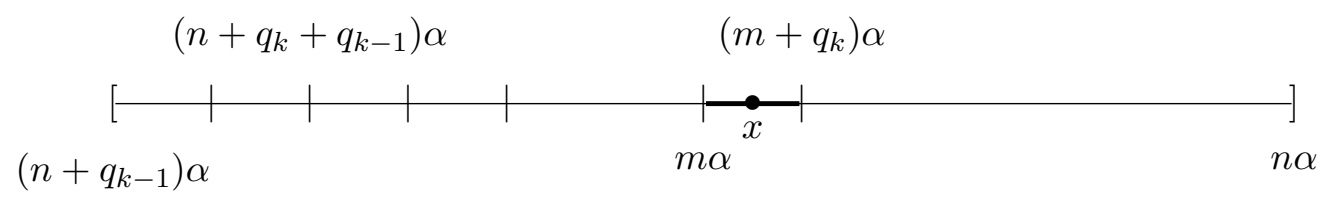

Figure 1. When $k$ is even, $x$ is in $I_{n}^{(k)} \cap I_{m}^{(k+1)}, m=b q_{k}+q_{k-1}+n$.

which attains the minimum $\left(2^{4} \cdot 3^{3}\right)^{-1}$ at $\delta=1 / 3$ and $M=72$. This completes the proof.

2.2. Non-singular on average. In this subsection, we show the implication (i) $\Longrightarrow$ (ii) of Theorem 1.1. Let us assume that $C_{0}:=\liminf _{k} \log q_{k} / k<\infty$ and show that $\operatorname{dim}_{H} \operatorname{Bad}^{\varepsilon}(\alpha)<1$. Since $\operatorname{Bad}^{\varepsilon}(\alpha)=\lim _{K \rightarrow \infty} \operatorname{Bad}_{K}^{\varepsilon}(\alpha)$, where

$$
\operatorname{Bad}_{K}^{\varepsilon}(\alpha):=\left\{x \in[0,1]:|q| \cdot\|q \alpha-x\| \geq \varepsilon, \forall q \geq q_{K}\right\}
$$

is an increasing sequence of sets, it is enough to show that $\operatorname{dim}_{H} \operatorname{Bad}_{K}^{\varepsilon}(\alpha)$ has a uniform upper bound smaller than 1 .

Throughout the paper, for a positive integer $n$, we view $n \alpha$ as a point on the circle $S^{1}=\mathbb{R} / \mathbb{Z}$ represented by an element in $[0,1)$. For convenience, given $a, b$ in $S^{1}$, we denote by $(a, b)$ the shorter interval among $(a, b)$ and $(b, a)$ in $S^{1}$ regardless of whether $a<b$ or not. For any $k$ in $\mathbb{Z}_{>0}$, define $\mathcal{P}^{(k)}$ to be the set of connected components of $S^{1} \backslash\left\{\alpha, 2 \alpha, \ldots, q_{k} \alpha\right\}$. We will call $\mathcal{P}^{(k)}$ a partition of $S^{1}$ by ignoring the endpoints of the intervals. This partition consists of $q_{k}$ intervals, which we call $I_{n}^{(k)}$, of the following two types :

(1) when $n<q_{k}-q_{k-1}: I_{n}^{(k)}=\left(n \alpha,\left(n+q_{k-1}\right) \alpha\right)$ and its length is $\left\|q_{k-1} \alpha\right\|$.

(2) when $n>q_{k}-q_{k-1}: I_{n}^{(k)}=\left(n \alpha,\left(n+q_{k-1}-q_{k}\right) \alpha\right)$ and its length is $\left\|q_{k-1} \alpha\right\|+\left\|q_{k} \alpha\right\|$. Their lengths are bounded as follows:

$$
\frac{1}{2 q_{k}}<\left\|q_{k-1} \alpha\right\|<\frac{1}{q_{k}} \text { and } \quad \frac{1}{q_{k}}<\left\|q_{k-1} \alpha\right\|+\left\|q_{k} \alpha\right\|<\frac{2}{q_{k}} .
$$

Let us denote by $|n|_{q}$ the integer in $[1, q]$ which is congruent to $n$ modulo $q$. With this notation, the elements of $\mathcal{P}^{(k)}$ are the intervals $I_{n}^{(k)}:=\left[n \alpha,\left|n+q_{k-1}\right|_{q_{k}} \alpha\right]$ with $n=1,2, \ldots, q_{k}$.

The partition $\mathcal{P}^{(k+1)}$ is clearly a refinement of the partition $\mathcal{P}^{(k)}$. Every element of $\mathcal{P}^{(k)}$ is divided into either $a_{k+1}$ or $a_{k+1}+1$ elements of $\mathcal{P}^{(k+1)}$. In particular, let $m$ be an integer with $q_{k}<m \leq q_{k+1}$, then $m \alpha$ is in $I_{n}^{(k)}$ if and only if

$$
m=n+q_{k-1}+c q_{k} .
$$

for some integer $0 \leq c \leq a_{k+1}-1$. See Figure 1,

We define a sequence $\left(k_{i}\right)_{i \geq 0}$ in the following way: set $k_{0}=K$ and, for $i \geq 0$, let $k_{i+1}$ be the smallest integer for which $q_{k_{i}} / q_{k_{i+1}}<\varepsilon / 12$. Observe that since $q_{k+2}>2 q_{k}$, the sequence $\left(k_{i+1}-k_{i}\right)_{i \geq 0}$ is uniformly bounded from above by a contant which we denote by $C_{\varepsilon}$. Hence we obtain

$$
\liminf _{i \rightarrow \infty} \frac{1}{i} \log q_{k_{i}}<C_{0} C_{\varepsilon}
$$

Note that

$$
\begin{aligned}
\operatorname{Bad}_{K}^{\varepsilon}(\alpha) & =\bigcap_{i=0}^{\infty} \bigcap_{q_{k_{i}} \leq n<q_{k_{i+1}}} B\left(n \alpha, \frac{\varepsilon}{n}\right)^{c} \subset \bigcap_{i=0}^{\infty} \bigcap_{q_{k_{i}} \leq n<\frac{\varepsilon}{2} q_{k_{i+1}}} B\left(n \alpha, \frac{\varepsilon}{n}\right)^{c} \\
& \subset \bigcap_{i=0}^{\infty} \bigcap_{q_{k_{i}} \leq n<\frac{\varepsilon}{2} q_{k_{i+1}}}\left(I_{n}^{k_{i+1}}\right)^{c} .
\end{aligned}
$$

The last inclusion above follows from the fact that each interval $I_{n}^{\left(k_{i+1}\right)}$ is contained in $B\left(n \alpha, \frac{\varepsilon}{n}\right)$ since it has one endpoint $n \alpha$ and is of length at most $2 / q_{k_{i+1}}<\varepsilon / n$. 
Thus, by letting $F_{K}=\bigcap_{i \geq 0} F_{K}^{(i)}$, where

$$
\mathcal{S}_{j}=\bigcap_{q_{k_{j}} \leq n<\frac{\varepsilon}{2} q_{k_{j+1}}}\left(I_{n}^{\left(k_{j+1}\right)}\right)^{c} \quad \text { and } \quad F_{K}^{(i)}=\bigcap_{0 \leq j \leq i} \mathcal{S}_{j},
$$

it is enough to find a uniform upper bound for $\operatorname{dim}_{H} F_{K}$.

We need the following lemma to estimate the number of subintervals of $I_{m}^{\left(k_{i+1}\right)}$ in each $I_{n}^{\left(k_{i}\right)}$.

Lemma 2.3. Let $Q \geq 6 q_{k}$. For each interval $I_{n}^{(k)}$ of $\mathcal{P}^{(k)}$, the number of points $m \alpha$ which belong to $I_{n}^{(k)}$ for $q_{k}<m \leq Q$ is at least equal to $Q / 4 q_{k}$.

Proof. Let $c$ be the positive integer defined by the inequalities

$$
q_{k}+c\left(2 q_{k}\right) \leq Q<q_{k}+(c+1)\left(2 q_{k}\right) .
$$

Each interval $I_{n}^{(k)}$ contains at least one point $m \alpha$ with

$$
(2 i-1) q_{k}+1 \leq m \leq(2 i+1) q_{k}, \quad 1 \leq i \leq c,
$$

since the length of interval $I_{n}^{(k)}$ is at least $\left\|q_{k-1} \alpha\right\|$ and the distance between two points of any neighboring point of $m \alpha$ with $(2 i-1) q_{k}+1 \leq m \leq(2 i+1) q_{k}$ is at most $\left\|q_{k-1} \alpha\right\|$.

Hence, the number of points $m \alpha$ contained in $\bar{I}_{n}^{(k)}$ with $q_{k}+1 \leq m \leq(2 c+1) q_{k} \leq Q$ is at least $c$. Using the assumption $Q \geq 6 q_{k}$, we have

$$
c>\frac{Q-3 q_{k}}{2 q_{k}} \geq \frac{Q}{4 q_{k}} .
$$

By Lemma 2.3, for each interval $I_{n}^{\left(k_{i}\right)}$ in $\mathcal{S}_{i}$, the number of intervals $I_{m}^{\left(k_{i+1}\right)}$ in $I_{n}^{\left(k_{i}\right)}$ which contain a point $m \alpha$ with $q_{k_{i}} \leq m \leq \varepsilon q_{k_{i+1}} / 2$ is at least $\varepsilon q_{k_{i+1}} /\left(16 q_{k_{i}}\right)$, since at most two points belong to one interval of $F_{K}^{(i+1)}$.

Since the total number of intervals $I_{m}^{\left(k_{i+1}\right)}$ contained in an interval of $I_{n}^{\left(k_{i}\right)}$ in $\mathcal{P}^{\left(k_{i}\right)}$ is at most

$$
\frac{\left\|q_{k_{i}-1} \alpha\right\|+\left\|q_{k_{i}} \alpha\right\|}{\left\|q_{k_{i+1}-1} \alpha\right\|}<\frac{4 q_{k_{i+1}}}{q_{k_{i}}}
$$

for each interval $I_{n}^{\left(k_{i}\right)}$ of $\mathcal{P}^{\left(k_{i}\right)}$, we have

$$
\frac{\#\left\{I_{m}^{\left(k_{i+1}\right)} \notin \mathcal{S}_{i+1}: I_{m}^{\left(k_{i+1}\right)} \subset I_{n}^{\left(k_{i}\right)}\right\}}{\#\left\{I_{m}^{\left(k_{i+1}\right)} \in \mathcal{P}^{\left(k_{i+1}\right)}: I_{m}^{\left(k_{i+1}\right)} \subset I_{n}^{\left(k_{i}\right)}\right\}}>\frac{\varepsilon q_{k_{i+1}} /\left(16 q_{k_{i}}\right)}{4 q_{k_{i+1}} / q_{k_{i}}}
$$

thus

$$
\frac{\#\left\{I_{m}^{\left(k_{i+1}\right)} \in \mathcal{S}_{i+1}: I_{m}^{\left(k_{i+1}\right)} \subset I_{n}^{\left(k_{i}\right)}\right\}}{\#\left\{I_{m}^{\left(k_{i+1}\right)} \in \mathcal{P}^{\left(k_{i+1}\right)}: I_{m}^{\left(k_{i+1}\right)} \subset I_{n}^{\left(k_{i}\right)}\right\}}<1-\frac{\varepsilon q_{k_{i+1}} /\left(16 q_{k_{i}}\right)}{4 q_{k_{i+1}} / q_{k_{i}}}=1-\frac{\varepsilon}{32} .
$$

Since the cardinality of $\mathcal{P}^{\left(k_{i}\right)}$ is $q_{k_{i}}$, the number of intervals $I_{n}^{\left(k_{i}\right)}$ in $\mathcal{S}_{i}$ is at most

$$
q_{k_{i}}\left(1-\frac{\varepsilon}{32}\right)^{i}
$$

Thus, for any $s \leq 1$, we have

$$
\sum_{I_{n}^{\left(k_{i}\right)} \in \mathcal{S}_{i}}\left|I_{n}^{\left(k_{i}\right)}\right|^{s}<q_{k_{i}}\left(1-\frac{\varepsilon}{32}\right)^{i} \cdot\left(\frac{2}{q_{k_{i}}}\right)^{s} .
$$


By (2.5), for any $M>C_{0} C_{\varepsilon}$, there exists a sequence $k_{i}$ tending to infinity for which $q_{k_{i}} \leq M^{i}$. Since $\bigcap_{k_{i}: q_{k_{i}} \leq M^{i}} F_{K}^{(i)}$ is a covering of $F_{K}$, we obtain

$$
\operatorname{dim}_{H} F_{K} \leq 1+\frac{\log (1-\varepsilon / 32)}{\log M} .
$$

This completes the proof of the lemma.

\section{Approximation of ONE-SIDED Limit}

In this section, we assume that the sequence $\left(a_{k}\right)_{k \geq 1}$ of partial quotients of $\alpha$ tends to infinity and prove Theorem 1.3. Recall that the partition $\mathcal{P}^{(k)}$ of $S^{1}$ consists of the intervals $I_{n}^{(k)}:=\left[n \alpha,\left|n+q_{k-1}\right|_{q_{k}} \alpha\right]$ with $n=1,2, \ldots, q_{k}$.

Recall also that the numbers $1 \leq m \leq q_{k+1}$ for which the corresponding point $m \alpha$ is contained in $I_{n}^{(k)}$ are (in order, looking from $\left|n+q_{k-1}\right|_{q_{k}} \alpha$ towards $\left.n \alpha\right) n+q_{k-1}+q_{k}, n+q_{k-1}+2 q_{k}$, etc.

If $I_{n}^{(k)} \supset I_{m}^{(k+1)}$ with $1 \leq n \leq q_{k}, 1 \leq m \leq q_{k+1}$, then we have

$$
m=n+q_{k-1}+b q_{k},
$$

for some integer $b$ with $-1 \leq b \leq a_{k+1}-1$. Thus two endpoints $m \alpha, n \alpha$ of $I_{m}^{(k+1)}, I_{n}^{(k)}$ are separated by

$$
\|m \alpha-n \alpha\|=\left\|\left(b q_{k}+q_{k-1}\right) \alpha\right\|=\left\|q_{k-1} \alpha\right\|-b\left\|q_{k} \alpha\right\| .
$$

To prove the first part of Theorem 1.3, let us fix $\varepsilon<1 / 4$. Choose $K$ large enough so that $a_{k}$ is large for every $k \geq K$ and select some sequence $\left(\gamma_{k}\right)_{k \geq 1}$ which tends to 0 as $k$ tends to infinity. We will later specify the conditions satisfied by $\gamma_{k}$.

Throughout this section, set $\delta_{k}:=\frac{q_{k-1}}{q_{k}}$. Since

and

$$
q_{k}\left\|q_{k} \alpha\right\|+q_{k}\left\|q_{k-1} \alpha\right\|=1+\left(q_{k}-q_{k-1}\right)\left\|q_{k} \alpha\right\|<1+\frac{q_{k} q_{k+1}\left\|q_{k} \alpha\right\|}{q_{k+1}}<1+\delta_{k+1}
$$

we get

$$
q_{k}\left\|q_{k-1} \alpha\right\|=1-q_{k-1}\left\|q_{k} \alpha\right\|>1-\frac{q_{k-1}}{q_{k}} \frac{q_{k}}{q_{k+1}} q_{k+1}\left\|q_{k} \alpha\right\|>1-\delta_{k} \delta_{k+1},
$$

$$
\left\|q_{k} \alpha\right\|+\left\|q_{k-1} \alpha\right\|<\frac{1+\delta_{k+1}}{q_{k}}, \quad\left\|q_{k-1} \alpha\right\|>\frac{1-\delta_{k} \delta_{k+1}}{q_{k}} .
$$

Lemma 3.1. Let $K$ be a natural number such that, for $k \geq K$, we have

$$
\gamma_{k}+2 \delta_{k}<1-2 \varepsilon^{1 / 2} .
$$

Let $k \geq K$. Then for every $n_{1}, n_{2}$ with

$$
\varepsilon^{1 / 2} q_{k}+q_{k-1}<n_{1}<\left(\varepsilon^{1 / 2}+\gamma_{k}\right) q_{k}+q_{k-1}
$$

and

$$
\varepsilon^{1 / 2} q_{k+1}+q_{k}<n_{2}<\left(\varepsilon^{1 / 2}+\gamma_{k+1}\right) q_{k+1}+q_{k},
$$

if $I_{n_{2}}^{(k+1)} \subset I_{n_{1}}^{(k)}$, then $I_{n_{2}}^{(k+1)}$ is disjoint from all the balls $B(n \alpha, \varepsilon / n)$ such that

(a) $n \alpha$ is an endpoint of $I_{n_{1}}^{(k)}$, or

(b) $q_{k}<n \leq q_{k+1}$ and $n \alpha$ is not an endpoint of $I_{n_{2}}^{(k+1)}$.

Proof. As $\left(n_{1}+q_{k-1}\right) \alpha$ and $\left(n_{2}+q_{k}\right) \alpha$ are endpoints of $I_{n_{1}}^{(k)}$ and $I_{n_{2}}^{(k+1)}$, for part (a), we need to check the inequalities

$$
\frac{\varepsilon}{n_{1}}<\left\|\left(n_{2}+q_{k}\right) \alpha-n_{1} \alpha\right\| \text { and } \frac{\varepsilon}{n_{1}+q_{k-1}}<\left\|n_{2} \alpha-\left(n_{1}+q_{k-1}\right) \alpha\right\| .
$$

Letting $n_{2}=b q_{k}+q_{k-1}+n_{1}$ as in (3.1), we have

$$
\left\|n_{2} \alpha-n_{1} \alpha\right\|=\left\|\left(b q_{k}+q_{k-1}\right) \alpha\right\|=\left\|q_{k-1} \alpha\right\|-b\left\|q_{k} \alpha\right\| .
$$


Therefore, we have

$$
\begin{aligned}
\left\|\left(n_{2}+q_{k}\right) \alpha-n_{1} \alpha\right\| & =\left\|q_{k-1} \alpha\right\|-(b+1)\left\|q_{k} \alpha\right\| \\
& =\frac{1-q_{k-1}\left\|q_{k} \alpha\right\|}{q_{k}}-\left(\frac{n_{2}-q_{k-1}-n_{1}+q_{k}}{q_{k}}\right)\left\|q_{k} \alpha\right\| \\
& >\frac{1}{q_{k}}-\left(\frac{n_{2}+\delta_{k+1} q_{k+1}}{q_{k}}\right)\left\|q_{k} \alpha\right\|>\frac{1-\varepsilon^{1 / 2}-\gamma_{k+1}-2 \delta_{k+1}}{q_{k}}>\frac{\varepsilon^{1 / 2}}{q_{k}},
\end{aligned}
$$

where the last inequality follows from (3.3). We also have

$$
\begin{aligned}
\left|n_{2} \alpha-\left(n_{1}+q_{k-1}\right) \alpha\right| & =b\left\|q_{k} \alpha\right\|=\frac{n_{2}-q_{k-1}-n_{1}}{q_{k}}\left\|q_{k} \alpha\right\| \\
& >\left(\frac{\varepsilon^{1 / 2} q_{k+1}+\left(1-\varepsilon^{1 / 2}-\gamma_{k}\right) q_{k}-2 q_{k-1}}{q_{k}}\right)\left(\frac{1-\delta_{k+1} \delta_{k+2}}{q_{k+1}}\right) \\
& >\frac{\varepsilon^{1 / 2}\left(1+\delta_{k+1}\right)\left(1-\delta_{k+1} \delta_{k+2}\right)}{q_{k}}>\frac{\varepsilon^{1 / 2}}{q_{k}} .
\end{aligned}
$$

Therefore, we have

$$
\frac{\varepsilon}{n_{1}}<\frac{\varepsilon}{\varepsilon^{1 / 2} q_{k}+q_{k-1}}<\frac{\varepsilon^{1 / 2}}{q_{k}}<\left|\left(n_{2}+q_{k}\right) \alpha-n_{1} \alpha\right|
$$

and

$$
\frac{\varepsilon}{n_{1}+q_{k-1}}<\frac{\varepsilon}{\varepsilon^{1 / 2} q_{k}+2 q_{k-1}}<\frac{\varepsilon^{1 / 2}}{q_{k}}<\left|n_{2} \alpha-\left(n_{1}+q_{k-1}\right) \alpha\right| .
$$

For part (b), we separate two cases:

(i) If $n \alpha$ is in $I_{n_{1}}^{(k)}$ and $n \alpha$ is not an endpoint of $I_{n_{2}}^{(k+1)}$, then $n=n_{2}-d q_{k}$ or $n=n_{2}+(d+1) q_{k}$ for some $d \geq 1$.

Suppose that $n=n_{2}-d q_{k}$. Then, by the condition $n>q_{k}$, we have

$$
1 \leq d<\frac{n_{2}}{q_{k}}-1
$$

Thus, combined with (3.4), we deduce that

$$
d\left(\frac{n_{2}}{q_{k}}-d\right) \geq\left(\frac{n_{2}}{q_{k}}-1\right)>\frac{\varepsilon^{1 / 2} q_{k+1}}{q_{k}}>\frac{2 \varepsilon q_{k+1}}{q_{k}}>\frac{\varepsilon}{q_{k}\left\|q_{k} \alpha\right\|} .
$$

Hence, for $n=n_{2}-d q_{k}$, we have

$$
\frac{\varepsilon}{n}=\frac{\varepsilon}{n_{2}-d q_{k}}<d\left\|q_{k} \alpha\right\|=\left|n \alpha-n_{2} \alpha\right| .
$$

When $n=n_{2}+d q_{k}$, we get

$$
\frac{\varepsilon}{n}=\frac{\varepsilon}{n_{2}+(d+1) q_{k}}<d\left\|q_{k} \alpha\right\|=\left|n \alpha-\left(n_{2}+q_{k}\right) \alpha\right| .
$$

(ii) If $n \alpha$ is not in $I_{n_{1}}^{(k)}$, then the distance between $n \alpha$ and $I_{n_{2}}^{(k)}$ is bigger than $\left|\left(n_{2}+q_{k}\right) \alpha-n_{1} \alpha\right|$ and $\left|n_{2} \alpha-\left(n_{1}+q_{k-1}\right) \alpha\right|$. By (3.5) and (3.6), we have

$$
\min \left\{\left|n \alpha-n_{2} \alpha\right|,\left|n \alpha-\left(n_{2}+q_{k}\right) \alpha\right|\right\}>\frac{\varepsilon^{1 / 2}}{q_{k}}>\frac{\varepsilon}{q_{k}}>\frac{\varepsilon}{n} .
$$

Denote by $F$ the set of all the points $x$ in $S^{1}$ such that, for all $k \geq K$, we have $x$ in $I_{n}^{(k)}$ with $\varepsilon^{1 / 2} q_{k}+q_{k-1}<n<\left(\varepsilon^{1 / 2}+\gamma_{k}\right) q_{k}+q_{k-1}$. By Lemma 3.1,

$$
\liminf _{n \rightarrow \infty} n\|n \alpha-x\| \geq \varepsilon
$$


Lemma 3.2. Under the assumptions of Lemma 3.1,

$$
I_{n_{2}}^{(k+1)} \subset B\left(\left(n_{1}+q_{k-1}\right) \alpha, \frac{\varepsilon_{k}}{n_{1}+q_{k-1}}\right),
$$

where $\varepsilon_{k}:=\left(\varepsilon^{1 / 2}+\gamma_{k}+2 \delta_{k}\right)\left(\varepsilon^{1 / 2}+\gamma_{k+1}+2 \delta_{k+1}\right)$.

Proof. Since $n_{2}=b q_{k}+q_{k-1}+n_{1}$, we have

$$
\begin{aligned}
\left|\left(n_{2}+q_{k}\right) \alpha-\left(n_{1}+q_{k-1}\right) \alpha\right| & =(b+1)\left\|q_{k} \alpha\right\|=\frac{n_{2}+q_{k}-q_{k-1}-n_{1}}{q_{k}}\left\|q_{k} \alpha\right\| \\
& <\left(\frac{\left(\varepsilon^{1 / 2}+\gamma_{k+1}\right) q_{k+1}+2 q_{k}}{q_{k}}\right)\left(\frac{1}{q_{k+1}}\right) \\
& =\frac{\varepsilon^{1 / 2}+\gamma_{k+1}+2 \delta_{k+1}}{q_{k}} \\
& =\frac{\varepsilon_{k}}{\left(\varepsilon^{1 / 2}+\gamma_{k}\right) q_{k}+2 q_{k-1}}<\frac{\varepsilon_{k}}{n_{1}+q_{k-1}} .
\end{aligned}
$$

Since $\gamma_{k}, \delta_{k}$ both tend to 0 as $k$ tends to infinity, the sequence $\left(\varepsilon_{k}\right)_{k \geq 1}$ tends to $\varepsilon$. Thus, Lemma 3.2 implies that

$$
\liminf _{n \rightarrow \infty} n\|n \alpha-x\| \leq \varepsilon
$$

By (3.8) and (3.7), the set $F$ is contained in $\left\{x: \liminf _{n} n\|n \alpha-x\|=\varepsilon\right\}$.

Lemma 3.3. If

then $\operatorname{dim}_{H} F=1$.

$$
\lim _{k \rightarrow \infty} \frac{\log \gamma_{k}}{\log a_{k}}=0
$$

Proof. Let

$$
F_{k}=\bigcup_{\varepsilon^{1 / 2} q_{k}+q_{k-1}<n<\left(\varepsilon^{1 / 2}+\gamma_{k}\right) q_{k}+q_{k-1}} I_{n}^{(k)} .
$$

Then $F=\cap_{k \geq K} F_{k}$. We may assume that $K$ is large enough to ensure that $\gamma_{k}>\delta_{k}$ for all $k \geq K$.

Each $F_{k}$ is a union of $q_{k-1}$ intervals of length at least $\left\lfloor\frac{\gamma_{k} q_{k}}{q_{k-1}}\right\rfloor\left\|q_{k-1} \alpha\right\|$ which are separated by at least

$$
\left\|q_{k-2}\right\|-\left\lceil\frac{\gamma_{k} q_{k}}{q_{k-1}}\right\rceil\left\|q_{k-1} \alpha\right\| .
$$

By Example 4.6. of [8], we obtain that

$$
\operatorname{dim}_{H} F \geq \liminf _{k \rightarrow \infty} \frac{\log \left(m_{K} m_{K+1} \cdots m_{k-1}\right)}{-\log \left(m_{k} \varepsilon_{k}\right)}
$$

where $m_{k}$ is the smallest number of intervals of $F_{k}$ in each interval of $F_{k-1}$ and $\varepsilon_{k}$ is the minimal the distance between intervals of $F_{k}$. Then we have

$$
m_{k}>\left(\frac{\gamma_{k-1} q_{k-1}}{q_{k-2}}-1\right)=\frac{q_{k-1}}{q_{k-2}}\left(\gamma_{k-1}-\delta_{k-1}\right), \quad m_{K}=q_{K-1},
$$

and

$$
\varepsilon_{k} \geq\left\|q_{k-2}\right\|-\left(\frac{\gamma_{k} q_{k}}{q_{k-1}}+1\right)\left\|q_{k-1} \alpha\right\| \geq\left(1-\gamma_{k}-\delta_{k}\right)\left\|q_{k-2} \alpha\right\|>\frac{1-\gamma_{k}-\delta_{k}}{2 q_{k-1}} .
$$

Since $\log \gamma_{k} / \log a_{k}$ goes to zero, we have

$$
\operatorname{dim}_{H} F \geq \liminf _{k \rightarrow \infty} \frac{\log q_{k-2}+\sum_{i=K}^{k-2} \log \left(\gamma_{i}-\delta_{i}\right)}{\log q_{k-2}-\log \gamma_{k-1}-\log \left(1-\gamma_{k}-\delta_{k}\right)+\log 2}=1
$$


Thus, the preceding three lemmas prove the first part of Theorem 1.3 ,

Now we prove the second part of Theorem 1.3 .

Lemma 3.4. Let $\varepsilon>1 / 4$. Then the set $\operatorname{Bad}_{+}^{\varepsilon}(\alpha)$ is empty.

Proof. Suppose that $\operatorname{Bad}_{+}^{\varepsilon}(\alpha)$ is nonempty and let $x$ be in this set. For any small positive $\delta$ with $\varepsilon-2 \delta>1 / 4$, we can choose $K$ large enough that $n\|n \alpha-x\| \geq \varepsilon-\delta$ for any $n>q_{K}$ and $\delta_{k+1}+\delta_{k+1}^{2}<\delta$ for $k \geq K$.

For $k=K, K+1, \ldots$, denote by $I_{n_{k}}^{(k)}$ the element of the partition $\mathcal{P}^{(k)}$ containing $x$.

For $n_{k+1}=n_{k}+c q_{k}+q_{k-1}$, the conditions $x \notin B\left(n_{k} \alpha,(\varepsilon-\delta) / n_{k}\right)$ and $x \in I_{n_{k+1}}^{(k+1)}$ imply that

$$
\begin{aligned}
\frac{\varepsilon-\delta}{n_{k}} & <\left|x-n_{k} \alpha\right| \leq\left|n_{k+1} \alpha-n_{k} \alpha\right|=\left|\left(c q_{k}+q_{k-1}\right) \alpha\right| \\
& =\left\|q_{k-1} \alpha\right\|-c\left\|q_{k} \alpha\right\|=\left(a_{k+1}-c\right)\left\|q_{k} \alpha\right\|+\left\|q_{k+1} \alpha\right\|<\frac{a_{k+1}-c}{q_{k+1}}+\frac{1}{q_{k+2}} .
\end{aligned}
$$

Therefore,

$$
n_{k+1}=n_{k}+c q_{k}+q_{k-1}<n_{k}+a_{k+1} q_{k}+q_{k-1}+\frac{q_{k} q_{k+1}}{q_{k+2}}-\frac{(\varepsilon-\delta) q_{k} q_{k+1}}{n_{k}}
$$

which implies

$$
\frac{n_{k+1}}{q_{k+1}}<1+\frac{n_{k}}{q_{k+1}}+\frac{q_{k}}{q_{k+2}}-\frac{(\varepsilon-\delta) q_{k}}{n_{k}}<1+2 \delta_{k+1}-(\varepsilon-\delta) \frac{q_{k}}{n_{k}}
$$

Therefore, for $k \geq K$, we get

$$
\begin{aligned}
\frac{n_{k}}{q_{k}}-\frac{n_{k+1}}{q_{k+1}} & >-1-2 \delta_{k+1}+(\varepsilon-\delta) \frac{q_{k}}{n_{k}}+\frac{n_{k}}{q_{k}} \\
& =\frac{q_{k}}{n_{k}}\left(\left(\frac{n_{k}}{q_{k}}\right)^{2}-\left(1+2 \delta_{k+1}\right) \frac{n_{k}}{q_{k}}+(\varepsilon-\delta)\right) \\
& =\frac{q_{k}}{n_{k}}\left(\left(\frac{n_{k}}{q_{k}}-\frac{1}{2}-\delta_{k+1}\right)^{2}+\varepsilon-\delta-\frac{1}{4}-\delta_{k+1}-\delta_{k+1}^{2}\right) \\
& \geq \varepsilon-2 \delta-\frac{1}{4}>0 .
\end{aligned}
$$

Thus, we have

$$
\frac{n_{k}}{q_{k}}<\frac{n_{K}}{q_{K}}-(k-K)\left(\varepsilon-2 \delta-\frac{1}{4}\right) \rightarrow-\infty \text { as } k \rightarrow \infty,
$$

which contradicts that $0<n_{k} / q_{k} \leq 1$.

\section{On REAL IRRATIONAL NUMBERS Which ARE SINGULAR ON AVERAGE}

In this section we consider the case $n=m=1$ and characterize the $1 \times 1$ matrices $(\alpha)$ which are singular on average, which gives the proof of the equivalence between (ii) and (iii) of Theorem 1.1 .

Proposition 4.1. Let $\alpha$ be a real number and $\left(p_{k} / q_{k}\right)_{k \geq 1}$ the sequence of its convergents. Then, $\alpha$ is singular on average if and only if $\left(q_{k}\right)^{1 / k}$ tends to infinity with $k$.

Proof. Let $0<c<1 / 2$ and let $k \geq 3$ be an integer. By the classical theory of continued fractions, we have

$$
\min _{0<n<q_{k+1}}\|n \alpha\|=\left\|q_{k} \alpha\right\| .
$$

Therefore, for each integer $X$ with $q_{k} \leq X<q_{k+1}$, the inequalities

$$
\|x \alpha\| \leq c X^{-1} \quad \begin{array}{ll}
\text { and } & 0<|x| \leq X \\
12 &
\end{array}
$$


have a solution if and only if $\left\|q_{k} \alpha\right\| \leq c X^{-1}$. Thus, for each integer $\ell$ in $\left[\log _{2} q_{k}, \log _{2} q_{k+1}\right)$ the inequalities (4.1) have no solutions for $X=2^{\ell}$ if and only if

$$
-\log _{2}\left(\left\|q_{k} \alpha\right\| / c\right)<\ell<\log _{2} q_{k+1} .
$$

Since $\left\|q_{k} \alpha\right\|<1 / q_{k+1}$, the number of integers $\ell$ in $\left[\log _{2} q_{k}, \log _{2} q_{k+1}\right)$ such that (4.1) have no solutions for $X=2^{\ell}$ is at most

$$
\left\lceil\log _{2} q_{k+1}+\log _{2}\left(\left\|q_{k} \alpha\right\| / c\right)\right\rceil<\log _{2} q_{k+1}+\log _{2}\left(\left\|q_{k} \alpha\right\| / c\right)+1<\log _{2}(1 / c)+1 .
$$

Hence, for an integer $N$ with $\log _{2} q_{k} \leq N<\log _{2} q_{k+1}$, the number of integers $\ell$ in $\{1, \ldots, N\}$ such that (4.1) have no solutions for $X=2^{\ell}$ is bounded from above by $\left(\log _{2}(1 / c)+1\right)(k+1)$, thus

$$
\begin{aligned}
& \frac{1}{N} \operatorname{Card}\left\{\ell \in\{1, \ldots, N\}: \text { inequalities (4.1) has no solution for } X=2^{\ell}\right\} \\
& \leq \leq \frac{\left(\log _{2}(1 / c)+1\right)(k+1)}{N} \leq \frac{\left(\log _{2}(1 / c)+1\right)(k+1)}{\log q_{k}},
\end{aligned}
$$

which converges to 0 as $k$ goes to infinity, as soon as $\left(q_{h}\right)^{1 / h}$ tends to infinity. Therefore, $\alpha$ is singular on average if $\left(q_{h}\right)^{1 / h}$ tends to infinity.

Suppose that $\alpha$ is singular on average. Choose $c=1 / 4$. Let $\ell$ be an integer satisfying $\log _{2} q_{k+1}-1 \leq \ell<\log _{2} q_{k+1}$ for some $k \geq 1$. Then, we have

$$
\left\|q_{k} \alpha\right\|>\frac{1}{2 q_{k+1}}=\frac{2 c}{q_{k+1}}>\frac{c}{2^{\ell}} .
$$

Since $\|n \alpha\| \geq\left\|q_{k} \alpha\right\|$ for any $0<n<q_{k+1}$, we conclude that (4.1) have no solutions for $X=2^{\ell}$ if $\ell$ is an integer in $\left[\log _{2} q_{k+1}-1, \log _{2} q_{k+1}\right)$. Recall that that $q_{k+1} \geq 2 q_{k-1}$, thus the intervals $\left[\log _{2} q_{k-1}-1, \log _{2} q_{k-1}\right)$ and $\left[\log _{2} q_{k+1}-1, \log _{2} q_{k+1}\right)$ are disjoint. Let $N$ be an integer with $\log _{2} q_{2 k} \leq N<\log _{2} q_{2(k+1)}$. Since the intervals

$$
\left[\log _{2} q_{2}-1, \log _{2} q_{2}\right),\left[\log _{2} q_{4}-1, \log _{2} q_{4}\right), \ldots,\left[\log _{2} q_{2 k}-1, \log _{2} q_{2 k}\right)
$$

are disjoint, the number of integers $\ell$ in $\{1, \ldots, N\}$ such that (4.1) have no solutions for $X=2^{\ell}$ and $c=1 / 4$ is at least $k$. Hence, we have

$$
\frac{k}{\log q_{2 k+2}} \leq \frac{k}{N}<\frac{1}{N} \operatorname{Card}\left\{\ell \in\{1, \cdots, N\}: \text { (4.1) have no solutions for } X=2^{\ell}, c=1 / 4\right\}
$$

and the condition of singularity on average implies that the right hand side of the inequality goes to 0 as $N$ goes to infinity. By the monotonicity of $\left(q_{k}\right)_{k \geq 1}$, we deduce that $\left(q_{k}\right)^{1 / k}$ goes to infinity.

\section{Proof of Theorem 1.5}

The key ingredient for the proof of Theorem 1.5 is the following statement.

Theorem 5.1. Let $n \geq 1$ be an integer. Let $\left(\mathbf{y}_{k}\right)_{k \geq 1}$ be a sequence of integer vectors such that $\left|\mathbf{y}_{k+1}\right| /\left|\mathbf{y}_{k}\right| \geq 4 n /(1-2 \delta)+1$ for $k \geq 1$, where $|\cdot|$ is the $L_{2}$-norm on $\mathbb{R}^{n}$. Assume that $\left|\mathbf{y}_{k}\right|^{1 / k}$ tends to infinity with $k$ (or some other suitable condition). Then, for any $\delta$ in $(0,1 / 2)$, setting $\mathcal{S}_{\delta}=\left\{\mathbf{x} \in[0,1]^{n}:\right.$ there exists $k_{0}(\mathbf{x})$ such that $\left\|y_{k, 1} x_{1}+\ldots+y_{k, n} x_{n}\right\|>\delta$ for all $\left.k \geq k_{0}(\mathbf{x})\right\}$, we have

$$
\operatorname{dim}_{H} \mathcal{S}_{\delta}=n .
$$

Proof. For $k \geq 1$ and $\delta$ in $(0,1 / 2)$, set

$$
E_{k, \delta}=\left\{\mathbf{x}=\left(x_{1}, \ldots, x_{n}\right) \in[0,1]^{n}:\left\|y_{k, 1} x_{1}+\ldots+y_{k, n} x_{n}\right\|>\delta\right\} .
$$

Let $h$ be an index such that

$$
\left|y_{k, h}\right|=\max _{\substack{1 \leq i \leq n \\ 13}}\left|y_{k, i}\right|
$$


For every $(n-1)$-tuple $\left(j_{1}, \ldots, j_{h-1}, j_{h+1}, \ldots, j_{n}\right)$ of integers from $\left\{0,1, \ldots,\left|y_{k, h}\right|-1\right\}$, there exist an integer $p$ and a real number $t$ with $0 \leq t<1 /\left|y_{k, h}\right|$, depending on $j_{1}, \ldots, j_{h-1}, j_{h+1}, \ldots, j_{n}$, such that

$$
y_{k, 1} \frac{j_{1}}{\left|y_{k, h}\right|}+\cdots+y_{k, h-1} \frac{j_{h-1}}{\left|y_{k, h}\right|}+y_{k, h} t+y_{k, h+1} \frac{j_{h+1}}{\left|y_{k, h}\right|}+\cdots+y_{k, n} \frac{j_{n}}{y_{k, h} \mid}=\frac{1}{2}+p .
$$

For each integer vector $\mathbf{j}=\left(j_{1}, \ldots, j_{n}\right)$ with $0 \leq j_{1}, \ldots, j_{n}<\left|\mathbf{y}_{k}^{(h)}\right|$, we write

$$
\mathbf{w}_{k}(\mathbf{j})=\left(\frac{j_{1}}{\left|y_{k, h}\right|}, \ldots, \frac{j_{h-1}}{\left|y_{k, h}\right|}, \frac{j_{h}}{\left|y_{k, h}\right|}+t, \frac{j_{h+1}}{\left|y_{k, h}\right|}, \ldots, \frac{j_{n}}{\left|y_{k, h}\right|}\right) \text {. }
$$

Then, there exists $\eta$ in $\{-1,1\}$ such that

$$
\left\|\mathbf{y}_{k} \cdot \mathbf{w}_{k}(\mathbf{j})\right\|=\left\|\eta j_{h}+p+\frac{1}{2}\right\|=\frac{1}{2} .
$$

For each $\mathbf{v}$ with $|\mathbf{v}|<\frac{1-2 \delta}{2\left|\mathbf{y}_{k}\right|}$, we have

$$
\left|\mathbf{y}_{k} \cdot \mathbf{v}\right| \leq\left|\mathbf{y}_{k}\right| \cdot|\mathbf{v}|<\frac{1}{2}-\delta .
$$

Therefore,

$$
\eta j_{h}+p+\delta<\mathbf{y}_{k} \cdot\left(\mathbf{w}_{k}(\mathbf{j})+\mathbf{v}\right)<\eta j_{h}+p+1-\delta,
$$

i.e.,

$$
\left\|\mathbf{y}_{k} \cdot\left(\mathbf{w}_{k}(\mathbf{j})+\mathbf{v}\right)\right\|>\delta .
$$

Let $B(\mathbf{w}, r)=\left\{\mathbf{v} \in \mathbb{R}^{n}:|\mathbf{w}-\mathbf{v}|<r\right\}$ be the ball centered at $\mathbf{v}$ of radius $r$ and set

$$
G_{k, \delta}:=\bigcup_{0 \leq j_{1}, \ldots, j_{n}<\left|\mathbf{y}_{k}\right|} B\left(\mathbf{w}_{k}(\mathbf{j}), \frac{1-2 \delta}{2\left|\mathbf{y}_{k}\right|}\right) .
$$

Then $G_{k, \delta}$ is contained in $E_{k, \delta}$. The balls composing $G_{k, \delta}$ are disjoint since

$$
\left|\mathbf{w}_{k}(\mathbf{j})-\mathbf{w}_{k}\left(\mathbf{j}^{\prime}\right)\right| \geq \frac{1}{\left|y_{k, h}\right|} \geq \frac{1}{\left|\mathbf{y}_{k}\right|}, \quad \text { for } \mathbf{j} \neq \mathbf{j}^{\prime}
$$

For $\left|\mathbf{j}-\mathbf{j}^{\prime}\right|_{\infty} \leq d$, with $d$ in $\mathbb{N}$, we have

$$
\left|\mathbf{w}_{k}(\mathbf{j})-\mathbf{w}_{k}\left(\mathbf{j}^{\prime}\right)\right| \leq \frac{\sqrt{n d^{2}+2 d+1}}{\left|y_{k, h}\right|} \leq \frac{\sqrt{n d^{2}+2 d+1}}{\left|\mathbf{y}_{k}\right| / \sqrt{n}} \leq \frac{n(d+1)}{\left|\mathbf{y}_{k}\right|} .
$$

Therefore, any ball of radius bigger than $\frac{n(d+1)}{2\left|\mathbf{y}_{k}\right|}+\frac{1-2 \delta}{2\left|\mathbf{y}_{k}\right|}$ contains $d^{n}$ balls of the form $B\left(\mathbf{w}_{k}(\mathbf{j}), \frac{1-2 \delta}{2\left|\mathbf{y}_{k}\right|}\right)$. Hence, each ball of $G_{k-1, \delta}$ contains at least

$$
\left(\frac{1-2 \delta}{n}\left(\frac{\left|\mathbf{y}_{k}\right|}{\left|\mathbf{y}_{k-1}\right|}-1\right)-2\right)^{n}
$$

balls of $G_{k, \delta}$. The condition $\left|\mathbf{y}_{k+1}\right| /\left|\mathbf{y}_{k}\right| \geq 4 n /(1-2 \delta)+1$ implies that

$$
m_{k} \geq\left(\frac{1-2 \delta}{n}\left(\frac{\left|\mathbf{y}_{k}\right|}{\left|\mathbf{y}_{k-1}\right|}-1\right)-2\right)^{n} \geq\left(\frac{2(1-2 \delta)}{1-2 \delta+4 n} \cdot \frac{\left|\mathbf{y}_{k}\right|}{\left|\mathbf{y}_{k-1}\right|}\right)^{n},
$$

where $m_{k}$ is a lower bound for the number of balls of level $k$ contained in a ball of level $\mathrm{i} k-1$. Any two balls are separated by at least $\varepsilon_{k}:=2 \delta /\left|\mathbf{y}_{k}\right|$. Putting $C=\left(\frac{2(1-2 \delta)}{1-2 \delta+4 n}\right)^{n}$, the mass distribution principle implies that

$$
\operatorname{dim}_{H} \mathcal{S}_{\delta} \geq \operatorname{dim}_{H}\left(\bigcap_{k} G_{k, \delta}\right)=\liminf _{k} \frac{\log \left(m_{1} \cdots m_{k-1}\right)}{-\log m_{k} \varepsilon_{k}^{n}}=\liminf _{k} \frac{n \log \left|\mathbf{y}_{k-1}\right|+k C}{\log \left|\mathbf{y}_{k-1}\right|}=n .
$$

This establishes the theorem. 
Proof of Theorem 1.5. We keep the notation from Subsection 1.2 and Theorem 1.5. In particular,

$$
\mathbf{y}_{k}={ }^{t}\left(y_{k, 1}, \ldots, y_{k, n}\right), \quad(k \geq 1),
$$

is a sequence of best approximation associated to the matrix $A$ and we set $Y_{k}:=\left|\mathbf{y}_{k}\right|$ for $k \geq 1$. We assume that the quotient $Y_{k+1} / Y_{k}$ tends to infinity with $k$. Let $\delta$ be in $(0,1 / 2)$. Let $\mathbf{x}$ be in $\mathcal{S}_{\delta}$, that is, such that

$$
\left\|y_{k, 1} x_{1}+\ldots+y_{k, n} x_{n}\right\| \geq \delta, \quad \text { for all } k \geq 1 .
$$

Let $\mathbf{q}$ be a non-zero integer $m$-tuple and let $k$ be the index defined by the inequalities

$$
Y_{k} \leq\left(2 m \delta^{-1}\right)^{m / n}|\mathbf{q}|^{m / n}<Y_{k+1} .
$$

Taking into account that $M\left(\mathbf{y}_{k}\right) \leq Y_{k+1}^{-n / m}$, the inequality (5.1) and

$$
\left\|y_{1} x_{1}+\cdots+y_{n} x_{n}\right\| \leq n|\mathbf{y}| \max _{1 \leq i \leq n}\left\|L_{i}(\mathbf{q})-x_{i}\right\|+m|\mathbf{q}| M(\mathbf{y})
$$

applied for $\mathbf{y}=\mathbf{y}_{k}$ give

$$
\delta \leq n Y_{k}\|A \mathbf{q}-\mathbf{x}\|+m|\mathbf{q}| Y_{k+1}^{-n / m}
$$

thus,

$$
\delta \leq n\left(2 m \delta^{-1}\right)^{m / n}|\mathbf{q}|^{m / n}\|A \mathbf{q}-\mathbf{x}\|+m\left(2 m \delta^{-1}\right)^{-1} .
$$

Consequently, we get

$$
\|A \mathbf{q}-\mathbf{x}\| \geq \frac{\delta}{2 n\left(2 m \delta^{-1}\right)^{m / n}}|\mathbf{q}|^{-m / n}
$$

By letting $\delta$ tend to $1 / 2$, this completes the proof of the second assertion of Theorem 1.5 .

For the first assertion, under the assumption that $Y_{k}^{1 / k}$ tends to infinity, we proceed as in the proof of Theorem 2.2 to extract a subsequence of $\left(\mathbf{y}_{\varphi(k)}\right)_{k \geq 1}$ of $\left(\mathbf{y}_{k}\right)_{k \geq 1}$ with the property that

$$
Y_{\varphi(k)} \geq R Y_{\varphi(k-1)}, \quad Y_{\varphi(k-1)+1} \geq Y_{\varphi(k)} / R, \quad \text { for } k \geq 2,
$$

where $R=4 n /(1-2 \delta)+1$ is given by Theorem 5.1. Then, everything goes exactly as above. We omit the details.

\section{ACKNOWLEDGEMENT}

DK is supported by the NRF of Korea (NRF-2015R1A2A2A01007090). SL is supported by Samsung Science and Technology Foundation under Project No. SSTF-BA1601-03. MR is supported by National Science Centre grant 2014/13/B/ST1/01033 (Poland).

\section{REFERENCES}

[1] Y. Bugeaud, Exponents of Diophantine approximation. In: Dynamics and Analytic Number Theory, 96-135, London Math. Soc. Lecture Note Ser., 437, Cambridge Univ. Press, Cambridge, 2016.

[2] Y. Bugeaud, S. Harrap, S. Kristensen, and S. Velani, On shrinking targets for $\mathbf{Z}^{m}$ actions on tori, Mathematika 56 (2010), 193-202.

[3] Y. Bugeaud and M. Laurent, Exponents of homogeneous and inhomogeneous Diophantine Approximation, Moscow Math. J. 5 (2005), 747-766.

[4] J. W. S. Cassels, An introduction to Diophantine Approximation, Cambridge Tracts in Math. and Math. Phys., vol. 99, Cambridge University Press, 1957.

[5] H. Davenport and W. M. Schmidt, Approximation to real numbers by algebraic integers, Acta Arith. 15 (1969), 393-416.

[6] M. Einsiedler and J. Tseng, Badly approximable systems of affine forms, fractals, and Schmidt games, J. Reine Angew. Math. 660 (2011), 83-97.

[7] P. Erdös and S. J. Taylor, On the set of points of convergence of a lacunary trigonometric series and the equidistribution properties of related sequences, Proc. London Math. Soc. 7 (1957), 598-615.

[8] K. Falconer, Fractal Geometry : Mathematical Foundations and Applications. John Wiley \& Sons, 1990.

[9] S. Kadyrov, D. Kleinbock, E. Lindenstrauss, and G. A. Margulis, Singular systems of linear forms and non-escape of mass in the space of lattices, J. Anal. Math. 133 (2017), 253-277.

[10] A. Ya. Khintchine, Über eine Klasse linearer diophantischer Approximationen, Rendiconti Circ. Mat. Palermo 50 (1926), 170-195. 
[11] A. Ya. Khintchine, Neuer Beweis und Verallgemeinerung eines Hurwitzschen Satzes, Math. Ann. 111 (1935), 631-637.

[12] A. Ya. Khintchine, Regular systems of linear equations and a general problem of Čebyšev, Izvestiya Akad. Nauk SSSR Ser. Mat. 12 (1948), 249-258.

[13] D. H. Kim, The shrinking target property of irrational rotations, Nonlinearity 20 (2007), 1637-1643.

[14] S. Lim, N. de Saxcé, and U. Shapira, Dimension bound for badly approximable grids, IMRN. To appear.

[15] H. Minkowski, Üeber die Annäherung an eine reelle Grösse durch rationale Zahlen, Math. Ann. 54 (1901), 91-124.

[16] N. G. Moshchevitin, A note on badly approximable affine forms and winning sets, Mosc. Math. J. 11 (2011), 129-137, 182.

[17] J. Tseng, Badly approximable affine forms and Schmidt games, J. Number Theory 129 (2009), 3020-3025.

IRMA, UMR 7501, Université de Strasbourg, CNRS, 7, Rue René Descartes, 67000 Strasbourg, FRANCE.bugeaud@math.unistra.fr

Department of Mathematics Education, Dongguk University - Seoul, 30 Pildong-Ro 1-Gil, JungGU, SeOul, 04620 Korea.kim2010@dongguk.edu

Department of Mathematical Sciences and Research Institute of Mathematics, Seoul National University, Kwanak-Ro, KwanaK-Gu, Seoul, Korea. slim@snu.ac.kr

Institute of Mathematics, Polish Academy of Sciences, ul. Śniadeckich 8, 00-656 Warszawa, POLAND.rams@impan.gov.pl 